\title{
Value of Serum miRNA-96-5p and miRNA-99a-5p as Diagnostic Biomarkers for Hepatocellular Carcinoma
}

\author{
Amal F Gharib (D)', Emad M Eed', Amany S Khalifa ${ }^{2}$, Nermin Raafat ${ }^{3}$, Somaia Shehab-Eldeen $\mathbb{D}^{4,5}$, \\ Hany R Alwakeel ${ }^{6}$, Ehab Darwiesh ${ }^{7}$, Abdallah Essa $\mathbb{D}^{4,5}$ \\ 'Department of Clinical Laboratories Sciences, College of Applied Medical Sciences, Taif University, Taif, 21944, Saudi Arabia; ${ }^{2}$ Clinical Pathology \\ Department, Faculty of Medicine, Menoufia University, Shebin El-Kom, Egypt; ${ }^{3}$ Department of Medical Biochemistry, Faculty of Medicine, Zagazig \\ University, Zagazig, Egypt; ${ }^{4}$ Tropical Medicine Department, Faculty of Medicine, Menoufia University, Shebin El-Kom, Egypt; ${ }^{5}$ Department of Internal \\ Medicine, College of Medicine, King Faisal University, Al-Ahsaa, Saudi Arabia; ${ }^{6}$ Department of Hepatology, National Liver Institute, Menoufia \\ University, Shebin El-Kom, Egypt; ${ }^{7}$ Department of Tropical Medicine, Faculty of Medicine, Zagazig University, Zagazig, Egypt
}

Correspondence: Somaia Shehab-Eldeen, Tropical Medicine Department, Faculty of Medicine, Menoufia University, Yassen Abd Al Ghafar Street, Shebin El-Kom, 325 I I, Egypt, Tel +20 II I725I523, Email somaia.shehab@med.menofia.edu.eg

Purpose: Circulatory microRNAs (miRNAs) have the potential to be employed as markers for cancer detection and as prognostic tools for disease management. As a result, our goal was to explore the effectiveness of serum miRNA-96-5p and miRNA-99a-5p as diagnostic tools in hepatocellular carcinoma (HCC).

Patients and methods: Blood samples were collected from 55 patients with HCV-induced HCC, 55 patients with HCV-induced liver cirrhosis, and 55 healthy controls. The expression levels of miRNA-96-5p and miRNA-99a-5p were measured using quantitative RT-PCR.

Results: miRNA-96-5p expression levels were increased in HCC patient sera, while miRNA-99a-5p levels were reduced. According to ROC curve analysis, using a combination of circulating miRNA-96-5p, miRNA-99a-5-, and alpha-fetoprotein (AFP) improves the accuracy of diagnoses for HCC, with an area under the curve (AUC) of 0.97 , compared to AUCs of $0.82,0.86$, and 0.73 , respectively, for the individual biomarkers. Furthermore, the present data suggested that higher serum miRNA-96-5p levels were linked to larger tumors and metastasis, whereas lower serum miRNA-99a-5p levels were exclusively linked to HCC metastasis.

Conclusion: Using miRNA-96-5p and miRNA-99a-5p in combination with AFP increased both sensitivity and specificity for the diagnosis of HCC. Furthermore, serum levels were linked to tumor size and metastasis. These findings suggested that serum miRNA96-5p and miRNA-99a-5p could be used as non-invasive biomarkers for the diagnosis of HCC.

Keywords: hepatocellular carcinoma, biomarkers, microRNA-96-5p, microRNA-99a-5p

\section{Introduction}

Hepatocellular carcinoma (HCC) is considered the most common primary hepatic malignancy. It is the sixth most frequent and the fourth most deadly type of cancer in the world. ${ }^{1,2} \mathrm{HCC}$ generally develops as a common sequel of liver cirrhosis. However, it is particularly linked to infection with hepatitis B (HBV) or hepatitis C (HCV) viruses and alcoholic or non-alcoholic fatty liver disease, which constitute the most common underlying etiologies of hepatic cirrhosis. $^{2}$ Despite recent advances in HCC prognosis, overall survival rates are still unsatisfactory, due to several factors. The poor prognosis for patients with HCC may be attributed largely to the lack of an effective means of early diagnosis. ${ }^{3}$ In general, the major diagnostic routes for HCC are based on histopathological examination, blood biomarkers, and imaging techniques. ${ }^{4}$ Serum alpha-fetoprotein (AFP) remains the most frequently used biomarker for HCC screening, early diagnosis, and evaluation of therapeutic efficacy. ${ }^{5}$

MicroRNAs (miRNAs) are small, non-coding, single-stranded RNA molecules (20-25 nucleotides), which can regulate gene expression through their effect on target messenger RNAs (mRNAs) at the post-transcriptional level. ${ }^{6}$ 
They have been found to be secreted into the extracellular environment and are able to circulate in various bodily fluids as part of extracellular vesicles (exosomes) or in association with circulating proteins. ${ }^{2}$ Dysregulated expression of miRNAs has been demonstrated in various cancers, including lung, prostate, colon, breast, and liver tumors, and has been shown to affect oncogene and tumor suppressor gene activity, thereby directly influencing carcinogenesis. ${ }^{7}$ Many miRNAs are critically involved in the processes of liver development, homeostasis, and pathophysiology. ${ }^{8}$

As a consequence of their dysregulated expression, circulating miRNAs have been studied as potential biomarkers for cancer, including HCC, and can be detected in serum or plasma using non-invasive techniques. ${ }^{2}$

MiRNA-96 was first discovered in the human cancer cell line HeLa in 2002 by Mourelatos et al as a member of the miR-183-96-182 cluster, located on human chromosome 7q32.2. ${ }^{9}$ Mature miRNA-96 can recognize and target mRNAs by complementary base pairing, resulting in silencing and/or degradation, depending on the degree of complementation. MiRNA-96 has been reported to act as an oncogenic miRNA, showing upregulated expression in various types of cancer and playing an important regulatory role in tumor pathogenesis by regulating target gene expression. ${ }^{10}$ Although its function in HCC is still unclear, ${ }^{11}$ miRNA-96 has been shown to be overexpressed in HCC cells, with the capacity to promote hepatic cell self-renewal and tumorigenicity. Moreover, many previous studies have revealed significant differences in miRNA-96 expression in hepato-carcinomatous and paracarcinomatous tissues. ${ }^{3}$

MiRNA-99a is a known clustered miRNA localized on human chromosome 21q21. In humans, miRNA-99a expression correlates negatively with adipose tissue inflammation and obesity. ${ }^{12}$ Several previous studies have reported downregulation of miRNA-99a in a wide range of human malignancies (ovarian carcinoma, bladder cancer, squamous cell carcinoma, prostate cancer, lung carcinoma, and hepatocellular carcinoma), suggesting a potential role for miRNA99a as a tumor suppressor. ${ }^{13-15}$ Furthermore, while miRNA-99a was found to be one of the most abundant microRNAs in normal human hepatic tissue, it was significantly downregulated in HCC. ${ }^{16}$ The aim of the current study was to investigate the potential role of miRNA-96-5p and miRNA-99a-5p as biomarkers for HCC.

\section{Subjects and Methods}

\section{Subjects}

A total of 165 participants were included in the current case-control study. They were included in the following groups: HCV-induced HCC, liver cirrhosis due to chronic hepatitis C, and age- and sex-matched healthy controls (each with 55 subjects). All of the patients were chosen from inpatient and outpatient clinics at the National Liver Institute, Menoufia governorate, Shebin Elkom, Egypt, from January 2020 to December 2020. All participants in this research underwent a clinical examination and had to provide a complete medical history. In addition, radiological investigations such as abdominal ultrasonography to assess hepatic focal lesions, liver cirrhosis, hepato-splenomegaly, ascites, and portal vein thrombosis, were performed. Patients with liver cirrhosis or HCC were diagnosed by clinical examination, laboratory tests for viral and tumor markers, abdominal ultrasound, and contrast-enhanced computed tomography. Tumors were staged using the Barcelona clinic liver cancer (BCLC) staging system. ${ }^{17}$ Patients were excluded from the study if they had liver disease due to causes other than viral hepatitis C, a history of prior treatment for HCV or HCC, or other malignant tumors. Furthermore, volunteers were excluded as healthy controls if they suffered from autoimmune diseases, severe liver or renal disease, hematologic disorders, or infectious diseases. All patients who were included in the study gave their informed consent. The ethical committee of the National Liver Institute of Menoufia approved the study, and it was carried out in line with the Helsinki Declaration of 1975.

\section{Methods}

Venous blood samples $(5 \mathrm{~mL})$ were taken in plain tubes under strict aseptic conditions and centrifuged for 10 minutes at $4^{\circ} \mathrm{C}$. The sera were separated and stored at $20^{\circ} \mathrm{C}$ until they were used. Another $2 \mathrm{~mL}$ of blood was taken in EDTA tubes for a complete blood count (CBC).

\section{Laboratory Investigations}

-Liver function tests: analyses of levels of enzymes, including alanine transaminase (ALT) and aspartate transaminase (AST), were performed on the automated AU480 chemistry analyzer (Beckman Coulter). 
-Serum alpha-fetoprotein (AFP): AFP levels in serum were estimated by a solid-phase, two-step chemiluminescence immunoassay using an Immulite 2000 system analyzer. The kits were supplied by Siemens (Siemens Healthineers, Malvern, USA). The normal AFP reference range was $0-20 \mathrm{ng} / \mathrm{mL}$.

-Hepatitis markers: anti-HCV antibodies and Hepatitis B surface antigen (HBsAg) were assayed by MEIA on an AxSYM automated analyzer, using kits supplied by Abbott (Abbott Laboratories, Illinois, USA).

-Analysis of serum miRNA-96 and miRNA-99a expression levels: total RNA was extracted from serum using the miRNeasy kit (Qiagen $\mathrm{GmbH}$ ), according to the manufacturer's instructions. To measure the quality of total RNA, the NanoDrop 2000 spectrophotometer (Thermo Fisher Scientific, Inc.) was used. RNA was stored at $-80^{\circ} \mathrm{C}$ for subsequent RT-qPCR analysis. The extracted RNA was reverse transcribed to synthesize cDNA using the miScript II RT kit (Qiagen $\mathrm{GmbH}$ ), in compliance with their manual.

The miScript SYBR Green PCR kit (Qiagen $\mathrm{GmbH}$ ) was used for real-time quantitative reverse transcription PCR (qRT-PCR), and reactions were carried out in duplicate, using the Stratagene Mx3005P-qPCR system. The endogenous control miR-16 was utilized to standardize the expression levels of miRNAs isolated from serum samples. ${ }^{18} \mathrm{PCR}$ conditions were as follows: initial denaturation at $95^{\circ} \mathrm{C}$ for 15 minutes, then 40 cycles of $94^{\circ} \mathrm{C}$ for 15 seconds, annealing at $55^{\circ} \mathrm{C}$ for 30 seconds, and extension at $70^{\circ} \mathrm{C}$ for 30 seconds. The primers were purchased from Qiagen GmbH. Primer sequences:

- MiRNA-96-5p forward, 5' TTGGGTGAAATATATTGTGCGTCTC 3';

- MiRNA-99a-5p forward, 5' GAGTCCTGGACACCCAACTACAAG 3';

- MiRNA-16 forward, 5' UAGCAGCACGUAAAUAUUGGCG 3';

- MiScript universal reverse primer, 5' AGCCGAAGTGAGCCACTGAA 3'.

Relative expression of the two miRNAs (miRNA-96-5p and miRNA-99a-5p) was calculated using the $\Delta \Delta C T$ method. ${ }^{19}$

\section{Statistical Analysis}

Results were analyzed using SPSS Statistics version 22. The data were expressed as percentage (\%), mean (x), standard deviation (SD). Association between qualitative variables was analyzed by the Chi-square test $(\chi 2)$. Student's $t$-test and Mann-Whitney test were used for comparison between two groups with quantitative variables showing normal and non-normal distribution, respectively. ANOVA was used to compare more than two groups with normally distributed quantitative variables. The Spearman correlation coefficient measured the degree of association between variables. ROC curve analysis was performed to evaluate the diagnostic performance of AFP, miRNA-96-5p, and miRNA-99a-5p. Finally, logistic regressions were used to assess the ability of combined tests to distinguish HCC from cirrhotic patients. P-values equal to or less than 0.05 were considered statistically significant.

\section{Results}

This study comprised 165 participants in three groups: 55 patients with HCC, 55 patients with liver cirrhosis, and 55 healthy individuals as controls. Their clinical and biochemical characteristics are shown in Table 1. There were no statistical differences between the three groups regarding age and sex. The HCC and cirrhotic cases showed significant statistical differences from controls in regard to AST, ALT, serum albumin, total bilirubin, international normalized ratio (INR), WBCs, hemoglobin, platelet count, AFP, miRNA-96, and miRNA-99a. HCC cases showed significant statistical differences compared with the cirrhotic group in regard to hemoglobin, AFP, miRNA-96-5p, and miRNA-99a-5p. Compared with cirrhotic patients, HCC patients had higher levels of miRNA-96-5p, while levels of miRNA-96-5p were also higher in cirrhotic patients than in controls. In contrast, levels of miRNA-99a-5p were significantly lower in the HCC group than the cirrhosis group, and significantly lower in the cirrhosis group than controls (Figure 1). Table 2 shows tumor characteristics in HCC patients. According to BCLC staging, 25 patients $(45.46 \%)$ were in stage A, 9 patients were in stage B (16.63), 18 patients $(32.73 \%)$ were in stage C, and 3 patients $(5.45 \%)$ were in stage D. As shown in Supplementary Table 1, there was no correlation between levels of miRNA-96-5p and miRNA-99a-5p and the laboratory results. Regarding the relationship between the two miRNAs under investigation and the clinicopathological 
Table I The Demographic and Laboratory Data of the Different Studied Groups

\begin{tabular}{|c|c|c|c|c|}
\hline $\begin{array}{l}\text { Variables } \\
\text { (Mean 士SD) }\end{array}$ & $\begin{array}{l}\text { HCC Group } \\
(n=55)\end{array}$ & $\begin{array}{l}\text { Cirrhosis Group } \\
(n=55)\end{array}$ & $\begin{array}{l}\text { Controls } \\
n=55\end{array}$ & $P$ value \\
\hline Age (years) & $54.83 \pm 6.54$ & $54.91 \pm 8.69$ & $55.40 \pm 7.24$ & 0.91 \\
\hline \multicolumn{5}{|l|}{$\operatorname{Sex}(N, \%)$} \\
\hline Male & $33(60.0)$ & $31(56.4)$ & $37(67.3)$ & 0.49 \\
\hline Female & $22(40.0)$ & $24(43.6)$ & $18(32.7)$ & \\
\hline \multicolumn{5}{|l|}{ Diabetes } \\
\hline Yes & $30(54.5)$ & $32(58.2)$ & - & 0.7 \\
\hline No & $25(45.4)$ & $23(41.8)$ & - & \\
\hline \multicolumn{5}{|l|}{ Child-Pugh score } \\
\hline Stage A & 39 (70.9l) & $33(60)$ & - & 0.531 \\
\hline Stage B & $12(21.82)$ & $15(27.3)$ & - & \\
\hline Stage C & $4(7.27)$ & $7(12.7)$ & - & \\
\hline ALT (U/L) & $80.56 \pm 27.48^{*}$ & $76.84 \pm 23.02 *$ & $33.27 \pm 14.84$ & $<0.001$ \\
\hline AST (U/L) & $85.95 \pm 24.59 *$ & $79.49 \pm 20.21^{*}$ & $22.78 \pm 8.43$ & $<0.001$ \\
\hline Albumin $(g / d l)$ & $3.31 \pm 0.2^{*}$ & $3.65 \pm 0.3^{*}$ & $4.32 \pm 0.4$ & $<0.001$ \\
\hline Total Bilirubin (mg/dl) & $1.36 \pm 0.24 *$ & $1.1 \pm 0.14^{*}$ & $0.85 \pm 0.07$ & $<0.001$ \\
\hline INR & $1.22 \pm 0.07^{*}$ & $1.17 \pm 0.04 *$ & $0.93 \pm 0.03$ & $<0.001$ \\
\hline WBCs $\left(\times 10^{9} / \mathrm{L}\right)$ & $6.42 \pm 2.86^{*}$ & $7.37 \pm 2.34^{*}$ & $7.72 \pm 2.62$ & 0.003 \\
\hline Hemoglobin (gm/dL) & $8.36 \pm 1.29^{*}, \#$ & $9.12 \pm 1.21^{*}$ & $12.29 \pm 1.73$ & $<0.001$ \\
\hline Platelet $\left(\times 10^{3} / \mu \mathrm{L}\right)$ & $138.75 \pm 33.22 *$ & $\left.|48.07 \pm 3| .2\right|^{*}$ & $273.51 \pm 64.95$ & $<0.001$ \\
\hline HCV RNA (IU/mL) & $397.907 \pm 1099.148$ & $103.898 \pm 230.092$ & - & 0.1438 \\
\hline Alpha-fetoprotein $(\mathrm{ng} / \mathrm{mL})$ & $211.13 \pm 165.52^{*}, \#$ & $79.09 \pm 43.46 *$ & $38.38 \pm 22.19$ & $<0.001$ \\
\hline miRNA-96-5p (RQ) & $1.92 \pm 0.80^{*}, \#$ & $0.89 \pm 0.61 *$ & $0.53 \pm 0.44$ & 0.001 \\
\hline miRNA-99a-5p (RQ) & $0.75 \pm 0.41^{*, \#}$ & $1.55 \pm 0.72^{*}$ & $2.65 \pm \mid .51$ & $<0.001$ \\
\hline
\end{tabular}

Notes: *Significantly differs from controls, " significantly differs from the cirrhotic cases.

Abbreviation: RQ; relative quantity.

characteristics of tumors in the HCC group, Table 3 shows that higher levels of serum miRNA-96-5p were associated with increased tumor size and metastasis, while lower levels of serum miRNA-99a-5p were associated with HCC metastasis only. ROC curve analysis was performed in order to determine the diagnostic usefulness of serum levels of

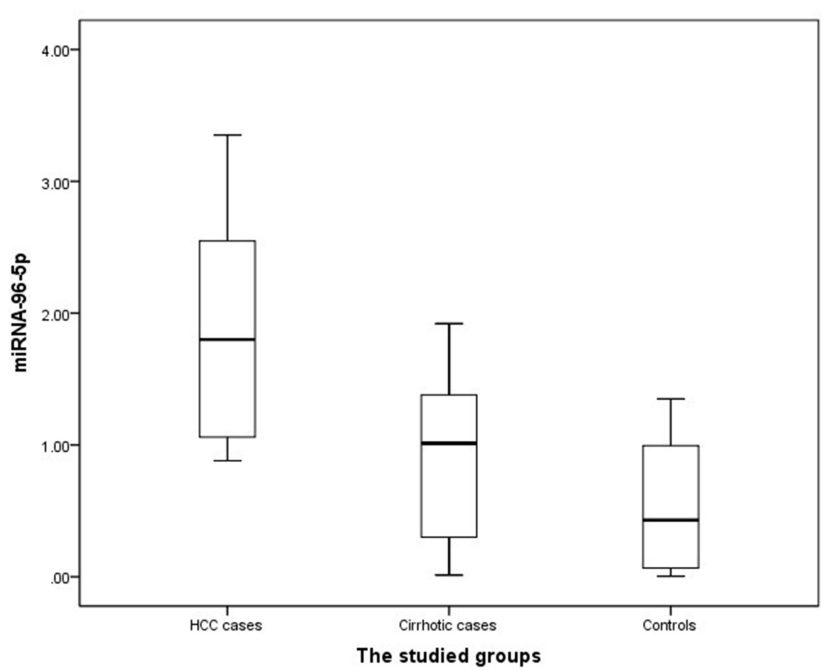

A

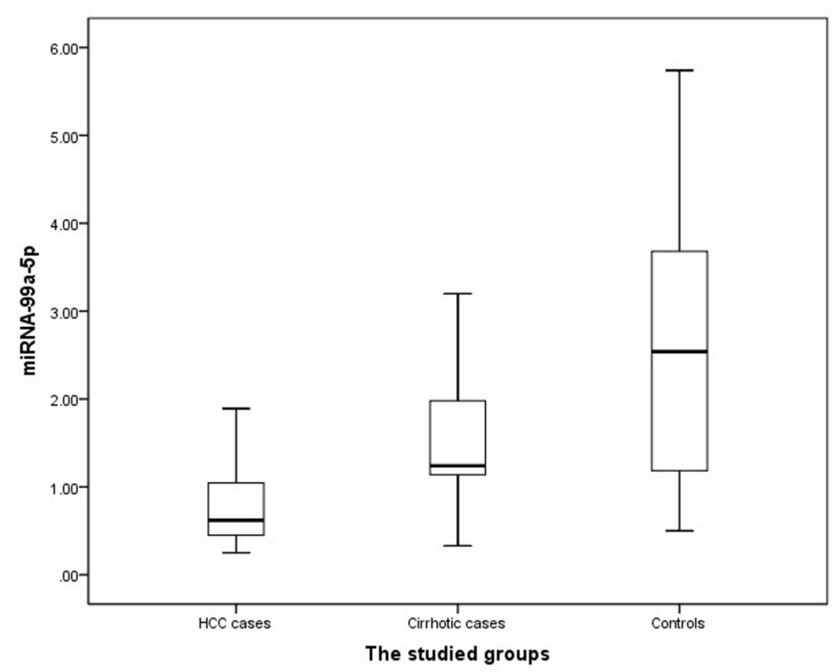

B

Figure I Comparison between (A) miRNA-96-5p, and (B) miRNA-99a-5p levels among the studied groups. 
Table 2 Tumor Characteristics in HCC Group

\begin{tabular}{|l|l|}
\hline Characteristics & No. (\%) \\
\hline $\begin{array}{l}\text { Focal lesion number } \\
\text { Single } \\
\text { multiple }\end{array}$ & $32(58.2)$ \\
& $23(41.8)$ \\
\hline $\begin{array}{l}\text { Tumor diameter } \\
<5 \mathrm{~cm}\end{array}$ & $29(52.7)$ \\
$\geq 5 \mathrm{~cm}$ & $26(47.3)$ \\
\hline PVT & $19(34.5)$ \\
Positive & $36(65.5)$ \\
Negative & \\
\hline Metastasis & $21(38.2)$ \\
Yes & $34(61.8)$ \\
No & \\
\hline BCLC stage & $25(45.46)$ \\
Stage A & $9(16.36)$ \\
Stage B & $18(32.73)$ \\
Stage C & $3(5.45)$ \\
Stage D & \\
\hline
\end{tabular}

Abbreviations: PVT, portal vein thrombosis; BCLC, Barcelona clinic liver cancer staging system.

Table 3 Relation Between miRNA-96-5p and miRNA-99a-5p and the Clinic-Pathological Characters Among HCC Group

\begin{tabular}{|c|c|c|c|c|}
\hline Character & $\begin{array}{l}\text { miRNA-96-5p } \\
X \pm S D\end{array}$ & P value & $\begin{array}{l}\text { miRNA -99a-5p } \\
X \pm S D\end{array}$ & $P$ value \\
\hline $\begin{array}{l}\text { Age (in years) } \\
\quad<55 \\
>55\end{array}$ & $\begin{array}{l}2.0 \pm 0.81 \\
I .84 \pm 0.80\end{array}$ & 0.44 & $\begin{array}{l}0.80 \pm 0.46 \\
0.69 \pm 0.36\end{array}$ & 0.33 \\
\hline $\begin{array}{l}\text { Tumor diameter } \\
\qquad 5 \mathrm{~mm} \\
\geq 5 \mathrm{~mm}\end{array}$ & $\begin{array}{l}1.72 \pm 0.72 \\
2.15 \pm 0.83\end{array}$ & $0.036 *$ & $\begin{array}{l}0.69 \pm 0.43 \\
0.81 \pm 0.39\end{array}$ & 0.09 \\
\hline $\begin{array}{l}\text { PVT } \\
\text { Positive } \\
\text { Negative }\end{array}$ & $\begin{array}{l}1.98 \pm 0.76 \\
1.88 \pm 0.83\end{array}$ & 0.65 & $\begin{array}{l}0.7 I \pm 0.39 \\
0.8 I \pm 0.45\end{array}$ & 0.42 \\
\hline $\begin{array}{l}\text { Metastasis } \\
\text { Yes } \\
\text { No }\end{array}$ & $\begin{array}{l}2 . \mid 7 \pm 0.81 \\
1.66 \pm 0.7 \mid\end{array}$ & $0.0175^{*}$ & $\begin{array}{l}0.57 \pm 0.23 \\
0.92 \pm 0.48\end{array}$ & $0.003 *$ \\
\hline $\begin{array}{c}\text { BCLC } \\
\text { A-B } \\
\text { C-D }\end{array}$ & $\begin{array}{l}1.80 \pm 0.78 \\
2.04 \pm 0.82\end{array}$ & 0.19 & $\begin{array}{l}0.78 \pm 0.42 \\
0.72 \pm 0.4 \mid\end{array}$ & 0.76 \\
\hline
\end{tabular}

Note: *Significant at $\mathrm{P}<0.05$.

miRNA-96-5p, miRNA-99a-5p, and AFP in HCC (Figure 2). For the three biomarkers analyzed, the area under the curve (AUC) of the ROC curve was as follows: AFP, 0.73; miRNA-96-5p, 0.82; and miRNA-99a-5p, 0.86. Combining the two miRNAs with AFP increased both the accuracy of diagnoses and the ability to discriminate between patients with HCC and patients with liver cirrhosis (Table 4). 


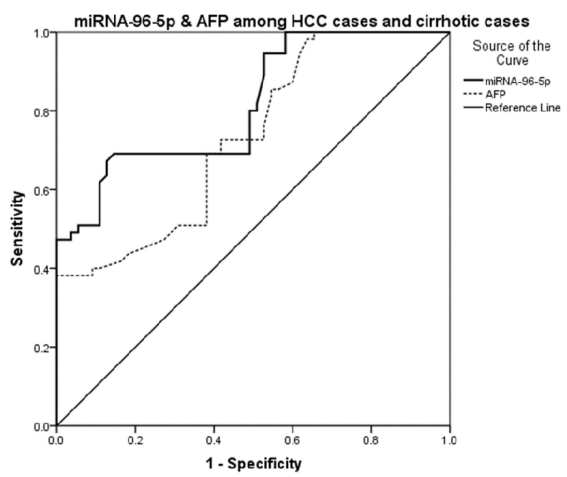

A

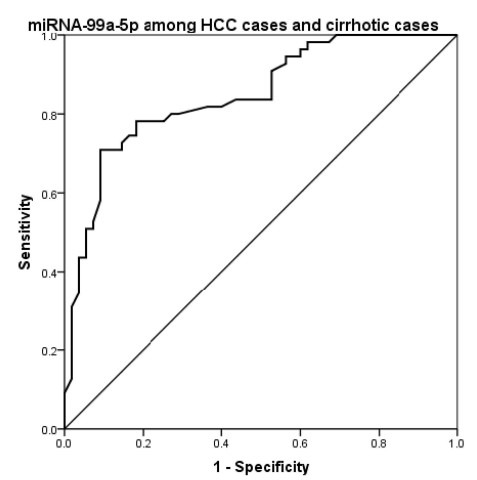

B

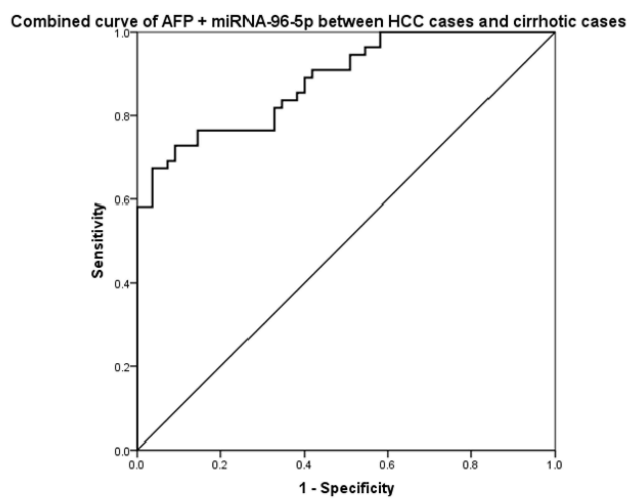

C

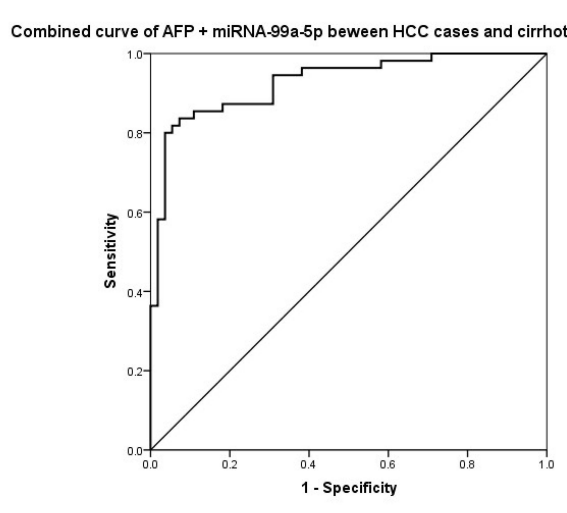

D

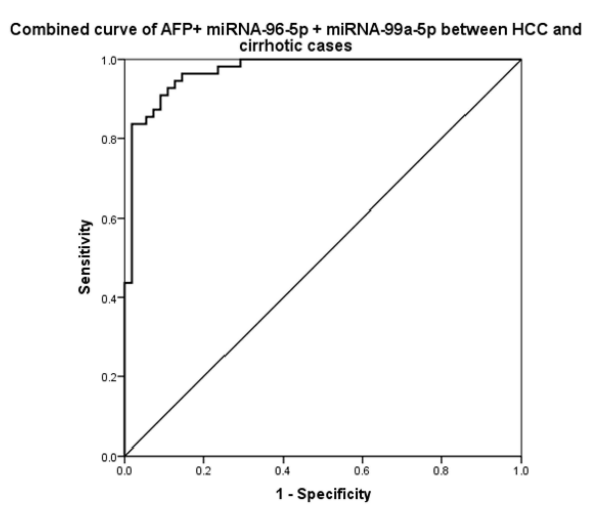

E

Figure 2 Receiver Operating Characteristic Curve (ROC) analysis of (A) AFP and miRNA-96-5p (B) miRNA-99a-5p (C) combined AFP + miRNA-96-5p (D) combined AFP + miRNA99a-5p (E) combined AFP + miRNA-96-5p + miRNA-99a-5p for distinguishing HCC from cirrhotic cases.

\section{Discussion}

Diagnosis of HCC is not possible using a single biomarker due to the complexity of its pathogenesis. Even AFP, the most informative marker, must be paired with positive disease-related radiological findings for diagnosis of $\mathrm{HCC}{ }^{20}$ With the recognition of miRNAs, a new horizon has opened up for research aimed at identifying new non-invasive markers for cancer detection. ${ }^{21}$ It is becoming increasingly clear that miRNAs influence several pathways within malignant tumors, including $\mathrm{HCC}^{22}$ and there has been evidence of miRNA deregulation in premalignant lesions, such as low-grade or high-grade dysplastic nodules, suggesting the involvement of miRNAs in early-stage HCC. ${ }^{23}$ Furthermore, miRNAs can regulate tumor progression and proliferation via acting as oncogenes or tumor suppressors. ${ }^{24}$

MiRNAs also play a significant role in many processes involved in HCV infection and hepatitis C. It is well known that MiRNA-122 is implicated in the HCV pathogenesis and lifecycle because it promotes the translation of viral proteins, stabilizes genomic RNA, and stimulates the replication of viral genomic RNAs. ${ }^{25,26}$ Moreover, targeting the HCV genome by interferon-induced miRNAs like miRNA-196 or miRNA-448 is believed to inhibit viral replication. ${ }^{27}$ Besides that, recent studies have highlighted the HCV protein's ability to modulate the expression of a host cell's miRNA, providing favorable conditions for the progression of chronic infection. ${ }^{28,29}$ The aim of this study was to assess the usefulness of serum levels of miRNA-96-5p and miRNA-99a-5p in the diagnosis of HCV-induced HCC.

Much research has suggested that abnormal miRNA-96 expression may play a crucial role in carcinogenesis and cancer progression, as observed for breast, stomach, lung, and bladder cancers. ${ }^{30-33}$ A previous study, conducted by Iwai et al, ${ }^{34}$ demonstrated that miRNA-96-5p expression levels were significantly higher in primary HCC tumors than in comparable non-tumorous tissues, suggesting a role in inhibition of apoptosis through targeting of caspase9 mRNA. In the current study, serum miRNA-96-5p levels were considerably higher in HCC cases than either cirrhotic patients or 
Table 4 Diagnostic Performance of Alpha-Fetoprotein, miRNA-96-5p, and miRNA-99a-5p for Distinguishing HCC Patients from Patients with Liver Cirrhosis

\begin{tabular}{|c|c|c|c|c|c|c|}
\hline & AFP & $\begin{array}{l}\text { miRNA- 96- } \\
5 p\end{array}$ & $\begin{array}{l}\text { miRNA-99a- } \\
\quad 5 p\end{array}$ & $\begin{array}{l}\text { Combined Both AFP\& miRNA- } \\
96-5 p\end{array}$ & $\begin{array}{l}\text { Combined Both AFP\& miRNA- } \\
\text { 99a-5p }\end{array}$ & $\begin{array}{l}\text { Combined AFP, miRNA-96-5p \& miRNA- } \\
\text { 99a-5p }\end{array}$ \\
\hline AUC & 0.73 & 0.82 & 0.86 & 0.88 & 0.95 & 0.97 \\
\hline $95 \% \mathrm{Cl}$ & $\begin{array}{c}0.64- \\
0.82\end{array}$ & $0.74-0.89$ & $0.78-0.92$ & $0.82-94$ & $0.92-0.98$ & $0.94-1.0$ \\
\hline $\begin{array}{l}\text { Cut off } \\
\text { point }\end{array}$ & 111.5 & 1.44 & 0.76 & - & - & - \\
\hline PPV & $63.4 \%$ & $82.6 \%$ & $88.9 \%$ & $88.9 \%$ & $92.2 \%$ & $94.0 \%$ \\
\hline NPV & $58.0 \%$ & $73.4 \%$ & $76.9 \%$ & $76.9 \%$ & $86.4 \%$ & $86.7 \%$ \\
\hline Accuracy & $60.0 \%$ & $77.3 \%$ & $81.8 \%$ & $81.8 \%$ & $89.1 \%$ & $90.0 \%$ \\
\hline$P$ value & $<0.001$ & $<0.001$ & $<0.001$ & $<0.001$ & $<0.001$ & $<0.001$ \\
\hline
\end{tabular}

Abbreviations: AUC, area under the curve; PPV, positive predictive value; NPV, negative predictive value. 
controls. Moreover, the levels in cirrhotic patients were significantly higher compared to controls, implying that miRNA96-5p overexpression may promote hepatic fibrosis and occur early in the development of HCC. Similar findings were reported by Chen et al. ${ }^{35}$ Another study reported upregulated expression of miRNA-96 in the sera of patients with HCC and showed that the upregulation was linked to shorter survival time. ${ }^{36}$ Regarding the correlation of miRNA-96-5p with the laboratory and clinicopathological characteristics of HCC, our results revealed a positive correlation with tumor size and metastasis. These results are in agreement with Chen et al, who found that high serum levels of miRNA-96 were associated with both larger tumor size and lymph node metastasis. ${ }^{35}$ However, in contrast to our results, they identified an association between increased levels of miRNA-96 levels and higher tumor stage. A possible explanation of this discrepancy may be the difference in the staging system used (TNM rather than BCLC).

The miRNA-99 family may act as tumor suppressors by affecting tumor cell proliferation, invasion, and migration. Downregulation has been reported in many cancers. ${ }^{37-40}$ MiRNA-99a is one of the most abundant miRNAs in normal liver tissue, and it is decreased significantly in HCC, suggesting a role in the development and progression of HCC. ${ }^{37}$ In the present study, serum levels of miRNA-99a-5p were significantly lower in HCC cases compared to cirrhotic patients and controls. Moreover, its levels in cirrhotic patients were significantly lower compared to controls, suggesting that downregulation of miRNA-99a-5p may be an early step in HCC development. This is in agreement with the results of a study conducted by Ning et $\mathrm{al}^{36}$ showing downregulation of miRNA-99a expression in tumor tissues and sera of HCC patients (compared to healthy controls), as well as with a number of other studies. ${ }^{37-39}$ As regards the correlation of miRNA-99a-5p with the laboratory and clinicopathological characteristics of HCC, our results revealed that lower levels of serum miRNA-99a were associated with metastasis. To date, no studies have evaluated the relationship between serum miRNA-99a and the clinicopathological features of the tumors in HCC. While Zhang et $\mathrm{al}^{40}$ found that lower levels of miRNA-99a expression in HCC tissues were related to low tumor differentiation, presence of liver cirrhosis, and overall survival, there was no correlation with tumor size, microvascular invasion, or tumor stage.

To evaluate the diagnostic and predictive value of serum miRNA-96-5p, miRNA-99a-5p, and AFP in distinguishing HCC patients from those with liver cirrhosis, ROC curve analysis was performed, and sensitivity, specificity, and area under the curve (AUC) were determined. The current study showed that, the AUC values for miRNA-96-5p and miRNA99a-5p were higher than the value for APF. Combining either miRNA with AFP also increased the AUC compared to AFP alone, while the highest diagnosis accuracy was achieved by combining both miRNAs with AFP. Therefore, our findings indicate that miRNA-96-5p and miRNA-99a-5p have a diagnostic role in HCC.

Our results were consistent with previous research showing a superior AUC for miRNA-96 (0.803) compared to AFP (0.713), at the cutoff point of 0.138 , and a further increase in AUC (to 0.889) when combining the two markers. ${ }^{35}$ Furthermore, another study showed AUCs of 0.824 and 0.799 for miRNA-96 and miRNA-99a, respectively. ${ }^{36}$

Our study has limitations due to the relatively small sample size, the focus on one ethnicity (Egyptians), and the fact that $\mathrm{HCV}$ represents the only risk factor for HCC. Hence, further large-scale, well-designed studies with diverse ethnicities and risk factors are needed to confirm the potential diagnostic and therapeutic role of these miRNAs.

\section{Conclusion}

The current study demonstrated that miRNA-96-5p expression was upregulated and miRNA-99a-5p expression was downregulated in the sera of HCC patients. Use of these miRNAs resulted in satisfactory diagnostic performance, while combining both miRNA-96-5p and miRNA-99a-5p with AFP resulted in further increased diagnostic accuracy for HCC, compared with using AFP alone. Furthermore, high serum levels of miRNA-96-5p were closely linked with large tumor size and metastasis, while low serum levels of miRNA-99a-5p were associated with metastasis. Collectively, these findings underlined the potential role of serum miRNA-96 and miRNA-99a-5p as diagnostic HCC biomarkers.

\section{Funding}

None.

\section{Disclosure}

The authors report no conflicts of interest in this work. 


\section{References}

1. Shi Y, Zhang -D-D, Liu J-B, et al. Comprehensive analysis to identify DLEU2L/TAOK1 axis as a prognostic biomarker in hepatocellular carcinoma. Mole Ther Nucl Acids. 2021;23:702-718.

2. Debes J, Romagnoli P, Prieto J, Arrese M, Mattos A, Boonstra A. Serum biomarkers for the prediction of hepatocellular carcinoma. Cancers. 2021;1681:2021.

3. Wang S, Yang Y, Sun L, Qiao G, Song Y, Liu B. Exosomal microRNAs as liquid biopsy biomarkers in hepatocellular carcinoma. Onco Targets Ther. 2021;2020:13.

4. Zhang N, Hu Z, Qiang Y, Zhu X. Circulating miR-130b-and miR-21-based diagnostic markers and therapeutic targets for hepatocellular carcinoma. Mol Genetics Genomic Med. 2019;7:e1012.

5. Wang T, Zhang K-H. New blood biomarkers for the diagnosis of AFP-negative hepatocellular carcinoma. Front Oncol. 2020;10:1316.

6. Ichiyama K, Dong C. The role of miR-183 cluster in immunity. Cancer Lett. 2019;443:108-114.

7. Ye J, Xu M, Tian X, Cai S, Zeng S. Research advances in the detection of miRNA. J Pharm Analysis. 2019;9:217-226.

8. Niveditha D, Jasoria M, Narayan J, et al. Common and unique microRNAs in multiple carcinomas regulate similar network of pathways to mediate cancer progression. Sci Rep. 2020;10:1-11.

9. Mourelatos Z, Dostie J, Paushkin S, et al. miRNPs: a novel class of ribonucleoproteins containing numerous microRNAs. Genes Dev. 2002;16:720-728.

10. Huang T, Fu Y, Wang S, et al. miR-96 acts as a tumor suppressor via targeting the BCR-ABL1 oncogene in chronic myeloid leukemia blastic transformation. Biomed Pharmacother. 2019;119:109413.

11. Huang Y, Zhang J, Li H, Peng H, Gu M, Wang H. miR-96 regulates liver tumor-initiating cells expansion by targeting TP53INP1 and predicts Sorafenib resistance. $J$ Cancer. 2020;11:6545.

12. Maurya M, Barthwal MK. MicroRNA-99a: a potential double-edged sword targeting macrophage inflammation and metabolism. Cell Mol Immunol. 2021;18:2290-2292.

13. Cui L, Zhou H, Zhao H, et al. Correction to: microRNA-99a induces G1-phase cell cycle arrest and suppresses tumorigenicity in renal cell carcinoma. BMC Cancer. 2021;21:1-2.

14. Wong T-S, Liu X-B, Wong BY-H, Ng RW-M, Yuen AP-W, Wei WI. Mature miR-184 as potential oncogenic microRNA of squamous cell carcinoma of tongue. Clin Cancer Res. 2008;14:2588-2592.

15. Gao W, Shen H, Liu L, Xu J, Xu J, Shu Y. MiR-21 overexpression in human primary squamous cell lung carcinoma is associated with poor patient prognosis. J Cancer Res Clin Oncol. 2011;137:557-566.

16. Eniafe J, Jiang S. MicroRNA-99 family in cancer and immunity. Wiley Interdiscip Rev RNA. 2021;12:e1635.

17. Llovet JM, Brú C, Bruix J. Prognosis of hepatocellular carcinoma: the BCLC staging classification. Semin Liver Dis. 1999;19:329-338. doi:10.1055/s-2007-1007122

18. Song J, Bai Z, Han W, et al. Identification of suitable reference genes for qPCR analysis of serum microRNA in gastric cancer patients. Dig Dis Sci. 2012;57:897-904.

19. Livak KJ, Schmittgen TD. Analysis of relative gene expression data using real-time quantitative PCR and the $2-\Delta \Delta C T$ method. methods. 2001;25:402-408.

20. Bruix J, Sherman M. Management of hepatocellular carcinoma: an update. Hepatology. 2011;53:1020-1022.

21. Hauptman N, Glavac D. MicroRNAs and long non-coding RNAs: prospects in diagnostics and therapy of cancer. Radiol Oncol. 2013;47:311.

22. Sadri nahand J, Bokharaei-Salim F, Salmaninejad A, et al. microRNAs: key players in virus-associated hepatocellular carcinoma. $J$ Cell Physiol. 2019;234:12188-12225.

23. Sulas P, Di Tommaso L, Novello C, et al. A large set of MiRNAs is dysregulated from the earliest steps of human hepatocellular carcinoma development. Am J Pathol. 2018;188:785-794.

24. Wong C-M, Tsang FH-C, Ng IO-L. Non-coding RNAs in hepatocellular carcinoma: molecular functions and pathological implications. Nat Rev Gastroenterol Hepatol. 2018;15:137-151.

25. Bandiera S, Pfeffer S, Baumert TF, Zeisel MB. miR-122-a key factor and therapeutic target in liver disease. J Hepatol. 2015;62:448-457.

26. Kunden RD, Khan JQ, Ghezelbash S, Wilson JA. The role of the liver-specific microRNA, miRNA-122 in the HCV replication cycle. Int J Mol Sci. 2020;21:5677.

27. Pedersen IM, Cheng G, Wieland S, et al. Interferon modulation of cellular microRNAs as an antiviral mechanism. Nature. 2007;449:919-922.

28. Kim G-W, Lee S-H, Cho H, Kim M, Shin E-C, Oh J-W. Hepatitis C virus core protein promotes miR-122 destabilization by inhibiting GLD-2. PLoS Pathog. 2016;12:e1005714.

29. Pascut D, Hoang M, Nguyen NN, Pratama MY, Tiribelli C, Proteins HCV. Modulate the host cell miRNA expression contributing to Hepatitis C pathogenesis and hepatocellular carcinoma development. Cancers. 2021;13:2485.

30. Lin H, Dai T, Xiong H, et al. Unregulated miR-96 induces cell proliferation in human breast cancer by downregulating transcriptional factor FOXO3a. PLoS One. 2010;5:e15797.

31. Tang X, Zheng D, Hu P, et al. Glycogen synthase kinase 3 beta inhibits microRNA-183-96-182 cluster via the $\beta$-Catenin/TCF/LEF-1 pathway in gastric cancer cells. Nucleic Acids Res. 2014;42:2988-2998.

32. Wu H, Zhou J, Mei S, et al. Circulating exosomal microRNA-96 promotes cell proliferation, migration and drug resistance by targeting LMO7. J Cell Mol Med. 2017;21:1228-1236.

33. Yoshino H, Seki N, Itesako T, Chiyomaru T, Nakagawa M, Enokida H. Aberrant expression of microRNAs in bladder cancer. Nat Rev Urol. 2013;10:396-404.

34. Iwai N, Yasui K, Tomie A, et al. Oncogenic miR-96-5p inhibits apoptosis by targeting the caspase-9 gene in hepatocellular carcinoma. Int J Oncol. 2018;53:237-245.

35. Chen Y, Dong X, Yu D, Wang X. Serum miR-96 is a promising biomarker for hepatocellular carcinoma in patients with chronic hepatitis B virus infection. Int J Clin Exp Med. 2015;8:18462.

36. Ning S, Liu H, Gao B, et al. miR-155, miR-96 and miR-99a as potential diagnostic and prognostic tools for the clinical management of hepatocellular carcinoma. Oncol Lett. 2019;18:3381-3387. 
37. Li D, Liu X, Lin L, et al. MicroRNA-99a inhibits hepatocellular carcinoma growth and correlates with prognosis of patients with hepatocellular carcinoma. J Biol Chem. 2011;286:36677-36685.

38. Yan B, Fu Q, Lai L, et al. Downregulation of microRNA 99a in oral squamous cell carcinomas contributes to the growth and survival of oral cancer cells. Mol Med Rep. 2012;6:675-681.

39. Yin H, Ma J, Chen L, et al. miR-99a enhances the radiation sensitivity of non-small cell lung cancer by targeting mTOR. Cell Physiol Biochem. 2018;46:471-481.

40. Zhang J, Jin H, Liu H, et al. MiRNA-99a directly regulates AGO2 through translational repression in hepatocellular carcinoma. Oncogenesis. 2014;3:e97-e97.

\section{Publish your work in this journal}

The International Journal of General Medicine is an international, peer-reviewed open-access journal that focuses on general and internal medicine, pathogenesis, epidemiology, diagnosis, monitoring and treatment protocols. The journal is characterized by the rapid reporting of reviews, original research and clinical studies across all disease areas. The manuscript management system is completely online and includes a very quick and fair peer-review system, which is all easy to use. Visit http://www.dovepress.com/testimonials.php to read real quotes from published authors.

Submit your manuscript here: https://www.dovepress.com/international-journal-of-general-medicine-journal 